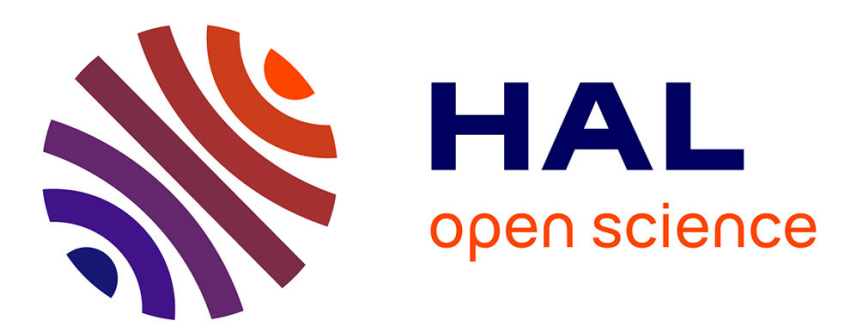

\title{
Le Paradoxe du Menteur: Essai de résolution dans le cadre d'une approche dynamique
}

Béatrice Godart-Wendling

\section{To cite this version:}

Béatrice Godart-Wendling. Le Paradoxe du Menteur: Essai de résolution dans le cadre d'une approche dynamique. Intellectica - La revue de l'Association pour la Recherche sur les sciences de la Cognition (ARCo), 1988, 6, pp. 123-168. hal-01314108

\section{HAL Id: hal-01314108 \\ https://hal.science/hal-01314108}

Submitted on 6 Mar 2017

HAL is a multi-disciplinary open access archive for the deposit and dissemination of scientific research documents, whether they are published or not. The documents may come from teaching and research institutions in France or abroad, or from public or private research centers.
L'archive ouverte pluridisciplinaire HAL, est destinée au dépôt et à la diffusion de documents scientifiques de niveau recherche, publiés ou non, émanant des établissements d'enseignement et de recherche français ou étrangers, des laboratoires publics ou privés. 


\title{
intellectica
}

\section{Langage et cognition}

\author{
J.-P. Desclés
}

B. Godart-Wendling, F. Rastier, R. Renaud, A. Trognon et Ch. Brassac, D. Vellard. 


\title{
Béatrice GODART-WENDLING
}

\author{
LE PARADOXE DU MENTEUR : ESSAI \\ DE RESOLUTION DANS LE CADRE \\ D'UNE APPROCHE DYNAMIQUE
}

Nous nous proposons de commencer cette étude par une présentation du paradoxe cou Menteur afin de mettre en évidence que ce paradoxe pent joler un rôle important dans l'ellaboration d'une théorie linguistiquze, car il possède non seulement une structure profondément dynamique mais est aussi un paradoxe sémantique très complexe à résoudre puisqùil pose à la fois des problèmes de signification, de référence et d'évaluation en texmes de vrai et faux.

Avant đe présenter l'intêrêt d'une résolution dynamique du paradoxe du Menteur, nous exposerons les principales méthodes de résolution (B. Russell, B. Van Fraassen, R.L. Martin et L. Goldstein) proposées dans le cadre d'une approche statique et nous montrerons que les enjeux dont est porteur le paradoxe du Menteur sont : la preuve de l'inconsistance du langage naturel et de son inéductibilité à un langage śormel ainsi que le problème de lincomplétrode du prédicat de vérité. Ceite présentation des différentes résolutions nous permettra d'une part de mettre en évidence que la cause de l'engendrement du paradoxe di Menteur n'a pas été véritablement décelée et d'autre part que la méthode de résolution adoptée est toujours externe et statique, c'est-à-dire qu'elle 
ne prend jamais en compte le dynamisme interne caractéristique du. paradoxe du Menteur. Il s'ensuit que dans cette approche de résolution, le paradoxe du Menteur conserve son statut d'énoncé problématique.

Nous proposerons alors une nouvelle approche de résolution dynamique et interne qui consistera à laisser librement évoluer l'engendrement du paradoxe dn Menteur, de telle façon qu'il deviendra possible de déterminer une procédure de résolution qui respecte l'évolution naturelle du paradoxe. La tésolution du paradoxe du Menterar autoréférent direct sera exposée, et celie-ci nous permettra de mettre en évidence que l'instabilité sémantique du paradoxe, créatrice de dynamisme, est due à une sous-détermination du contenu propositionnel de l'énoncé paradoxal.

\section{Présentation du paraloxe du Menteur}

La notion de paradoxe a été longtemps réservée au domaine de Ia Logique. Toutefois, nous nous proposons de montrer, grâce à l'étude dar paradoxe du Menteur, que cette notion possède aussi un domaine d'application en Linguistique, et tout particulièrement dans le champ de 1a Sémantique.

Le paradoxe du Menteur s'Enonce ainsi : cet énoncé est faux et appartient à la famille des paradoxes Sui-falsificateurs, car il a pour propriété đe référer systématiquement à lui-mồme (sui-), en indiquant qu'il est faux (falsificateur).

Ce paradoxe donne lieu à l'apparition du raisonnement suivant : $\mathrm{s}$ 'il est vrai que cet énoncé est faux, alors cet énoncé est faux. Mais cet énoncé indiquait de hui-même qu'il était faux, donc il disait la vérité. Donc $€ e ̂$ énoncé est vrai. Mais, si cet énoncé est ఛrai, alors il est foux, puiscqu'il dit de lui-mêtme, non qa'il est vrai, mais qu'il est faux...

Nous pouvons qualifier ce raisonnement de têduction à l'absurde ou de régression infinie, car l'alternance "Cet énoncé est faux / Cet énoncé est 
rrait" peut être poursuivie indéfiniment. Il est anssi possible de parler de "cercle vicienx", car le raisonnement s'effectue toujours grâce au passage de "Cet énoncé est faux" à "Cet énoncé est vrai" et vice-versa.

Considérons maintenant quelles notions sémantiques sont concernées par l'existence du paradoxe du Menteur.

Nous pouvons tout d'abord constater qu'il remet en cause les concepts sémantiques de Vérité et de Fausseté, puisqu'il pose le problème de son évaluation en termes de vrai et de faux, pour une théorie lingristique qui utilise une logique vériconditionnelle. En effet, comment peut-on attribuer une valeur de vérité à un énoncé qui se caractérise par le fait d'être altemativement vrai et faux ?

Le paradoxe du Mentewr pose aussi le problème de la détermination de la référence de son terme sujet : ainsi, si l'on recherche le référent sousjacent à "Cet énoncé", on obtient toujours une réponse peu satisfaisante, car renvoyant à l'énoncé de départ : "c'est : cet énoncé est faux". La question se pose alors de savoir si "cet énoncé" est un terme singulier référentiel ou un terme à référent vide.

De plus, il pose le problème de la détemination de sa signification, s'il est lu (comme il le préconise de lui-même) en lecture "réflexîu"e", c'està-dire au sens de : "L'ênoncé que je suis en train de produire, d'écrire...." En effet, si cet énoncé signifie qu'il est faux, alors il se présente tel ca'ii est et signiffe donc qu'il est vrai. Il s'ensuit que le paradoxe du Menteur pose le problème Iinguistique du passage par quasi-transitivité de la signification de l'énoncé ("Cet énoncé est faux") vers sa signification inverse ("Cet énoncé est vrai"). Le paradoxe đu Menteur a donc pour propriété remarquable dobtenir une structure contradictoire de façon profondément dynamique, puisque le changement s'effectue, indéfiniment et naturellement, par étapes discontinues entre un énoncé faux et un énoncé vrai. De plus, le paradoxe du Menteur serable mettre en jeu un raisonnement complet, au sens où aucune prémisse supplémentaire r'est exigée pour que la dynamique interne, caractéristique de la présence 
du raisonnement, ait lieu. Le problème du passage par quasi-transitivité de la signifrication serait donc révélateur đu fait que ce paradoxe repose sur un raisonnernent complet.

Le paradoze du Menteur pose aussi deux problèmes attenants au champ ce la pragmatique : on peut tout d'abord se demander s'il ne viole pas les maximes de quartitité et de qualité de P. Grice. En effet pent-on estimer que le locuteur qui a dit : "Cet énoncé est faux" a foumi le maximun des informations en sa possession ? et ceci d'autant plus qu'il ne précise pas quel est le téférent de "cet énoncé"...

De même, la présomption de vérité (maxime de qualité) a-t-elle été respectée par celui qui dit cet éroncé est fawn, c'est-à-dire je mens? Le second problème pourrait presque être appelé un problème pragnnatique par đéfaut. II concexne le fait que l'énoncé cet énoncé est faux possède la propriété remarquable de đevenir paradoxal dès qu'il est prononcé par un locuteur, et ceci indépendamment de.la personnalité du locuteur.

\section{Rappel des différentes résolutions du paradoxe du Menteur dans le cadre d'une approche statique}

Avant de présenter l'intérêt d'une résolution dynamique du Paradoxe du Menteur, nous allons exposer les principales méthodes de résolution proposées dans le cadre d'une approche statique. Cette présentation nous permettra de mettre en évidence les difficultés que l'on rencontre lorsque l'approche de résolution choisie n'est pas conforme à la nature du problème que l'on a à résoudre.

Ainsi, l'étude des différentes résolutions du Paradoxe du Menteur, proposées de l'antiquité au XXe siècle, nous a permis de considérer qu'il y a trne convergence tant sur la cause de l'engendrement de ce paradoxe (régression infinie / cercle vicieux / contradiction) que sur les métbodes à utiliser pour le résoudre (création de métalangage ê de concepts opératoires). 
En effet, que ce soit en logique vériconditionnelle d'ordre deux ou multi-valuée, đeux voies de résolution pervent être dégagées :

- On élabore un nouveau systèmue logistique ( La Théorie des Types pour B. Russełl en logique bivalente ou la Théorie des Super-évalnations de B. Van Fraassen en logique trivalente) exprimable grâce à la création d'un langage formel, qui permet de sinnuler la production de l'énonoé du. Menteur et de le rescuüre; on

- On recherche à l'intérieur même du langage naturel les concepts aptes à résondre le paradoxe (La Théorie des Catégories Sémantiques de R.L. Martin et L. Goldstein ).

Nous allons maintenant exposer brièvement ces deux voies de résolution

\section{II.1. Résolution du paradoxe du Menteur dans un langage formel}

\section{II.1.1. La résolution de B. Russell en logique bivalente : la théorie des} Types

Pour B. Russell, la cause de l'engendrement du paradoxe do Menteur est due à la présence dun cercle vicieux. En effet, Ia sui-référence qui porte sur le terme sujet de l'énoncé paradoxal a pour conséquence que ce terme ne pent se déterminer que par rapport au tout qu'il détermine. Il s'ensuit qu'un cercle vicieux est créé : dans l'énoncé do Menteur, la variable "Cet énoncé" prend pour valeur cette même variable.

En fonction de cette analyse, B. Russell va élaborer le principe du cercle vicieux : Tout ce qui contient une variable apparente ne doit pas être une valeur possible de cette variable, qui pose que toute réflexivité est interdite en Langue. II s'ensuit qu'mn énoncé ne peut plus dire de luimême qu'il est vrai ou faux, c'est-à-dire qu'il ne peut plus s'attribuer sa propre valeur de vérité. 
Le principe du cercle viciexx, empêche donc lengendrement du paradoxe du Menteur, en prohibant dès le niveau de la Langue, la róflexivité. Le bien-fondé de ce principe provient du fait qu'il sanvegarde la Langue de toute inconsistance, puigu'elle riest plus apte à engendrer des contradictions telles que le paradoze du Merteur (Cet énoncé est faux si et seulement si il est vrai). Cependant, B. Russell note (1906, p - 640-41) : "1l importe de remarquer que le principe du cercle vicieux I'est pas lui-même la solution des paradoxes de cercle vicieux, mais seulement la conséquence qu'une théorie doit fournir pour apporter une solution. Autrement dit, il faut construire une thécrie des expressions contenant des variables apparentes qui foumisse comme conséquence le principe du cercle vicieux. Cest pour cette raison que nous avons besoin d'une reconstruction des premiers principes logiques, et que nous ne pouvons pas nous contenter de ce simple fait que les paradoxes sont dus à des cercles vicieux".

B. Russell va alors élaborer un nouveau système logistique binaire, la Théorie des Types, qui constime le cadre théorique explicatif des paradoxes sui-falsificateurs.

La base de la thécrie des types est de réduire les énoncés à des fonctions propositionnelles. Une fonction propositionnelle aura la fomme " $\mathrm{x}$ est un homme", on "sin $x=1$ ", c'est-à-dire qu'elle contient une variable $x$, et n'exprime une proposition que si une valeur est assignée à $x$. A chaque fonction propositionnelle correspond "in domaine de signification" constiture par les arguments pour lesquels cette fonction peut prendre des valeurs. Ainsi la fonction est vraie ou fansse, lorsquelle prend pour arguments ceux qui appartiennent à son domaine de signification; et elle est sans signification sì les arguments n'appartiennent pas à son domaine de siguification.

B. Russell écrit ainsi : "La notion de type est définie comme le domaine de signification a'tune fonction propositionnelle, c'est-à-dire comme l'ensemble des arguments pour lesquels la dite fonction a des valeurs. A 
chaque fois qu'ane variable apparente apparaît dans une proposition, le domaine des valeurs de la variable apparente est un type, le type étant déterminé par la fonction dont "toutes les valeurs" sont concerrées. La division des objets en types est imposée par les faux raisonnements reflexif̂s qui autrement surviennent" (1908, p. 75).

Les différents types de propositions forment une hiérarchie cont le principe inducteur est qu'une fonction propositionnelle qui contient tne variable apparente doit être d'm type inméciatement supérieur à toutes Ies valeurs possibles de cette variable. Les variables apparentes ont done pour rôle de đéterminer le type de la fonction.

La théorie hiérarchisée des types se présente done ainsi :

- Le premier type est constitué par les termes des propositions élémentaires, qui sont appelés les individus. Exemple : une pomme. Le premier type permet de générer le type suṕrieur, en effectuant un processus de généralisation sur les individus, de façon à ce qu'ils deviennent des variables apparentes. Ainsi :

- Le deuxième type est constitué des propositions élémentaires qui contiement comme variables apparentes des individus. Les propositions du deuxieme type seront par exemple: "la pomme est rouge". Ces propositions sont appelées propositions du premier ordre. Cet ensemble de propositions va permettre de générer à son tour le type supérieur. En effet, nons pouvons former de nouvelles propositions, qui anront pour variables apparentes les propositions du premier ordre. Ainsi :

- Le troisième type est constitué des propositions du second oräte, qui ont pour variables apparentes les propositions da premier ordre. Ainsi la proposition : "I'énoncé de Paul : «la pomme estrouge» est yrai" est une proposition du second ordre.

Le troisième type possède donc, entre autres, un prédicat de vétité, car il doit être capable de đéterminer la valeur de vérité des propositions du premier ordre. Le deuxième type n'a pas de prédicat de vézité ou de fausseté, car on ne peut attribuer une valeur de vérité à des individus 
( *la pomme est wraie). La đétermination de la valeur de vérité d'une proposition, dans la théorie des types, s'effectue donc toujours grâce au type supérieur (excepté pour le premier type).

L'engendrement des types supétieurs an troisième type se poursuit de façon inductive, de telle sorte que le quatrième type est constitué des propositions du troisième ordre, qui ont pour variables apparentes les propositions du second ordre... B. Russell résume ainsi le principe d'induction (1908, p. 7677) : "le nième +1 type logique sera constitué de propositions d'ordre $n$, qui seront telles qu'elles contiendront des propositions d'ordre $\mathrm{II}-1$, mais pas d'un ordre plus élevé, comme variabies apparentes. Les types ainsi obtemus sont muluellement exclusifs, et ainsi aucune erreur refflexive n'est possible tant que nous nous souvenons quine variable apparente doit toujouts être confinée à lintétieur d'un quelconque type déterminé".

La détermination de la valeur de vérité des propositions de chaque type d'ordre $n>2$ est effectuée par le type imméđiatement supérieur, de même que le troisièrme type attribuait une valeur de vérité aux propasitions du deuxième type. Chaque type $(n>2)$ possède donc son propre prédicat de vérité qui détermine la valeur de vérité des propositions du type strictement inférieur. Cette hiérarchie des prédicats de vérite dans la théorie des Types est due au fait que B. Russefl estime que les prédicats de vérité, tels qu'ils sont employés en langage ordinaire, sont ambigus.

Aussi, dans la theorie des Types, le langage naturel se constitue en fonction dune hiérarchie de langages allant dn type 1 an type n, telle qu'à aucun niveau de la hiérarchie un énoncé ne peut dire de lui-même qu'il est vrai ou faux. Ainsi un énoncé d'ordre $m$, contenant les termes "vrai $i^{m}$ ou "faux $\mathrm{wa}_{\text {" }}$ ne sera jamais vrai ${ }_{\mathrm{m}}$ ou faux $\mathrm{m}$, mais cun đegré de vérité ou de fausseté durn type toujours superieur à m (c'est-à-dire $: m+1$ ). La therorie des Types, en fournissant une structure logique du langage 
naturel, où la réflexivité n'est plus exprimable, fournit donc une base théorique au principe du cercle vicieux.

La résolution du paradoxe du Menteur, dans le cadre de la théorie des Types, se présente donc ainsi :

L'énonce "Je mens" peut être interpreté comme signifiant : $l$ y a une proposition que j'affirme et qui est fausse. La forme propositionnelle conespondant à cet énoncé est : J'asserte p, et $p$ est fraux, où $p$ est la variable apparente. Or, nous avons vu que pour $B$. Russell, le prédicat de vérité ou de fausseté đu langage ordinaire est ambigu. Nous devons donc lever lambiguinté du predicat "est faux", en spécifiant le type auquel il appartient dans la hiérarchie des types. Ceci revient à déterminer le type de la proposition du Menterr. Ainsi, si nous posons que la proposition $p$ da Menteur se sitze à lordre n (type $n+1$ ), étant donné le principe inducteur de la thérie des Types, la proposition qui contient $p$ comme variable apparente doit appartenir à l'ordre immédiatement supérieur, done à $\mathrm{I}+1$ (type $\mathrm{n}+2$ ). Donc, l'ordre du prédicat de fausseté contentz dans l'énoncé : Il y a une proposition $p$ que j'affirme et qui a une fausseté d"ordre $n$, doit être d'un orüre supérieur à n, c'est-à-dire $\mathrm{n}+1$ (type $\mathrm{n}+2$ ). B. Russell résume ainsi cette résolution $(1943 / 69, \mathrm{p} .75)$ : "Lhomme qui dit : "je vous raconte un mensonge d'ordre $\mathrm{n}^{\text {" }}$ vous raconte vraiment un mensonge, mais d'ordre $\mathrm{n}+1 . " \mathrm{La}$ theorie hiêrarchisée des Types, en riayant pas la propriété d'être réflexive, puisque chaque type détermine la valeur de vérité des propositions du type inférièer, empeche que l'attribution de la valeur de véritê de $\mathrm{p}$ se fasse au même type que $\mathrm{p}$, et donc empêche l'apparition de la contradiction.

Pour B. Russell, le paradoxe du Menteur, tel qu'il est habituellement formulé, pose donc le problème de l'inconsistence du langage naturel. Il s'ensuit que Ie paradoxe du Menteur constituerait la preuve de lirréductibihté du langage naturel à un langage formel. Il devient par conséquent nécessaire de montrer, grâce à la théorie des Types, que 
ce paradoxe est syntaxiquement mal formé, puisqu'il prédique đe lui-même sa propre fansseté.

Dans cente approche, un enjen du paradoxe du Menteur est donc la possibilité de la réflexivité en Langue. Cet enjeu est imaportant, car de nombreux énoncés du langage naturel sont réflexifs. Ex. : Cet énoncé est composé de mots français qui est, pour $\mathrm{B}$. Russell, syntaxiquement mal formé.

I1.1.2. La résolution de B. Van Fraassen en logique trivalente: la théorie des Super-êvaluations

B. Van Fraassen a repris l'analyse fatte par P.F. Strawson qui consiste à dire que l'énoncé du Menteur souffire d'un échec presuppositionnel. En effet, lorsqu'un locuteur entend l'énoncé du Menterr, il décèle, de par la présence du pronom démonstratif et du terone singulier Cet énoncé que l'existence de "Cet énoncé" a été présupposée par le locuteur. Il se trouve alors placé dans la situation de trouver à quel énoncé "Cet énoncé" réfêre. Mais sa recherche va se révéler vaine, car lorsqu'il se posera la question : "Quel est cet énoncé ?", il obtiencira torjours un énoncé en tout point identique à celui qui a été prononcé par le Mentenr. C'est : "cet enoncé est faux". Par conséquent, la cause de l'engendrement du paradoxe dn Menteur est une régression infinie composée de l'énoncé du Menteur lui-même qui a pour conséquence que le terme sujet sonffre dun échec présuppositionnel existentiel.

Aussi, B. Van Fraassen va commencer son étude du paradoxe du Menteur par la recherche d'uze définition précise de la relation de Présupposition. Cette définition permettra de mettre en évidence la spécificité de la relation de présupposition du paradoxe du Menteur. Il deviendra alors possible de construire un langage formel qui sera apte à exprimer le paradoxe et à le résoudre. 
La relation de Présupposition entre les phrases est ainsi définie par B. Van Fraassen :

(1) A présuppose $\mathrm{B}$ si et seulement si A est ni vraie ni fausse à moins que B soit vraie. (1968, p. 137)

La définition (1) peut encore se réécrire:

(2) A présuppose B si et seulement si :

(a) si A est vraie alors B est vraie.

(b) si (non-A) est vraie alors $B$ est vraie.

Où la négation doit être interprété comme une négation de choix, c'està-đire : (non-A) est vraie si et seulement si A est fausse. La définition (2) peut encore être précisée si l'on fait appel à la relation sếmantique de Contrainte qui se définit ainsi :

(3) A contraint B si et seulement si, toutes les fois que A est vraie, B est aussi vraie (1968, p. 138)

La relation de présupposition se compose donc d'une double relation de Contrainte : nous pouvons en effet constater, grâce à la définition (2) que non seulement $A$ contraint $B$, mais (non-A) contraint aussi $B$.

Les définitions (2) et (3) vont alors permettre à B. Van Fraassen d'obtenir une nouvelle définition de la présupposition, qui fait intervenir la relation de contrainte:

(4) A présuppose B si et seulement si :
(a) A contraint $B$
(b) (non-A) contraint $B$

La relation sémantique de présupposition entre des phrases $A$ et $B$ ayant été spécifiée grâce à la relation plus primaire de contrainte, il devient nécessaire de construire un langage formel qui aura les moyens 
d'exprimer la relation de présupposition. En effet, ce langage sera apte à engendrer un énoncé type Menteur et une résolution pourra alors être proposée.

Cependant, ce langage se doit aussi de rendre compte du fait que le paradoxe du Menteur est un cas limite pour le prédicat đe vérité, an sens où cet énoncé est vrai à la condition qu'il soit faux. ll s'ensuit que lenjen dont est portelue le paradoxe du Menteur, pour B. Van Fraassen, est de poser le problème de lincomplêtudo du prédicat de vérité ou de fausseté, puisque ce paradoxe indique que nos règles héritées de la logique bivalente et qui sont censées régir la structure du langage naturel sont inadéquates pour déterminer la valeur de vérité de l'ênoncé du Menteur.

La démarche que B. Van Fraassen va donc suivre est de créer une extension de la logique bivalente, de telle façon que les énoncés qui peuvent être évaluês par la logique bivalente en termes de "vrai" ou "faux" conservent leur évaluation classique; les autres énoncés (les cas limites entre autres) se verront évalués en termes de "vrai", "faux" ou "ni yrai ni faux" en conformité avec les tables de vérité correspondantes1. Cette extension de la logique classicque est appelée ta théorie des superévaluations.

La notion de super-évaluation est ainsi définie par B. Van Fraassen (1968, p. 142) :

La super-êvaluation induite par l'ensemble $G$ des phrases vraies dans une situation donnée est la fonction qui :

(a) assigne Vrai à A si toutes les évaluations classiques qui satisfont $\mathrm{G}$ assignent Vrai à A.

(b) assigne Faux à A si toutes les évaluations classiques qui satisfont $\mathrm{G}$ assignent Faux à $\mathrm{A}$.

${ }^{1}$ Ce. pour les tables de vérité : McCAWLEY, J.D., 1981, pp. 244-245. 
En conséquence, toutes les phrases qui ont pu être correctement évaluées en logique bivaiente conservent leur valeur de vérité dans la theorie des Super-évaluations

(c) n'est pas défimie pour $\mathrm{A}$, autrement (c'est-à-đire ni vrai nł faux).

Dans la sémantique de B. Van Fraassen une condition minimale est posée sur le prédicat de verité : elle exige simplement que lorsque A est vraie, V(A) le soit aussi, et inversement.

Ceci revient à dire que $A$ et $V(A)$ se contraignent mutrellement :

- A contraint V(A); et

- V(A) contraint A.

B. Van Fraassen interprète donc la convention (T) de A.Tarski :

$\mathrm{V}(\mathrm{A})$ si et seulement si $\mathrm{A}$

selon la relation de Contrainte et non comme exprimant une relation d'Implication.

De plus, contrairement aे B. Russell , B. Van Fraassen pense qu'il n'y a "aucune raison a priori" qui pourrait empêcher un énoncé d'être suiréflexif par rapport à lui-même ou à un de ses termes. B. Van Fraassen distingue alors deux cas de suj-référence pouvant être tencontrés dans le langage naturel. I oppose ainsi la sui-référence accidentelle à la suiréférence fonctionnelle et les définit de la façon suivante (1970, p. 426):

"Toutes les fois que la sui-référence est́ la référence à une plurase aur moyen diun terme survenant dans cette phrase (que ce soit un nom, une description, ou un prédicat), et que ce terme est tel que toutes ses occurrences ont la même extension, nous parlerons de sui-référence accidentelle". L'Enoncé «la neige est blanche» est une phrase, est un exemple de swi-référence accidentelle, car «la neige est blanche» est une phrase est dans l'extension de est une phrase, et ce dernier terme a toujours la même extension. Par contre «Quand la sui-référence est 
prodnitte à travers l'usage de termes dont la référence est dépendante du contexte, nous parlerons de sui-référence fonctionnelle».

La sui-reférence présente dans l'énonce du Menteut est une suiréférence fonctionnelle, car elle provient de l'usage de cette "phrase" dont la référence "Cette phrase est fausse" ne peot être déterminée que par rapport au contexte. B. Van Fraassen analyse alors la nature de la relation que cette sui-référence fonctionnelle entretient avec la pinrase $X$ du Menteur Cette phrase est fausse comme étant une relation sémantique de Contrainte :

$\mathrm{X}$ contraint $\mathrm{V}(-\mathrm{X})$; et

$\sim X$ contraint $V(\sim X)$.

Ce quỉ, d’aprés la définition (4), est équivalent à :

$\mathrm{X}$ présuppose $\mathrm{V}(-\mathrm{X})$.

Or, nous savons que $V(\sim X)$ ne peut être vraie, puisque la condition minimale qui a été posśe sur le prédicat de vérité est que $X$ et $V(X)$ se contraignent mułuellement. $\mathrm{X}$ a donc une présupposition qui n'est pas vraie, ce qui a pour conséquence (cf. définition (1)) que $X$ est ni vraie ni fausse. La phrase dur Menteur souffre de l'échec d'une relation logique qui lui est interne : la relation de présupposition.

L'échec de la relation logique de présupposition, décelé par B. Van Fraassen, à l'intérieur de la phrase du Menteur, pourrait encore être exprimé en disant que $\mathrm{X}$ est ni vraie ni fausse, car elle présuppose une contradiction. En effet, d'après la condition minimale imposée sur le prédicat de vérité :

$\mathrm{X}$ contraint $\mathrm{V}(\mathrm{X})$

Or gous venons de voir que :

$X$ contraint $V(-X)$

Donc $X$ présuppose une contradiction. 
Le caractère non-trivial de la relation de présupposition contenue dants le paradoxe du Menteur peut maintenant être explicité : nous savons que $X$ ne présuppose pas $V(X)$, puisque $\sim X$ ne contraint pas $V(X)$, mais $V(-X)$. Par contre, nous venons de voir que $X$ contraint $V(X)$ et que $(-X)$ contraint $V(\sim X)$. Donc $X$ et $\sim X$ contraignent à la fois $V(X) v V(\sim X)$. Donc $X$ présuppose sa propre bivalence. La présupposition inteme à la phrase du Menteur est donc un cas limite de présupposition.

En conclusion, l'enjeu dont est porteur le paradoxe du Menteur pour B. Van Fraassen, en étant un cas limite de présupposition, est l'incomplétude du prédicat de vérité. D'où la mise en oeuvre d'une logique multi-valuée.

L'approche de B. Russell et de B. Van Fraassen consiste donc à munir un langage formel de moyens techniques qui lui pernettent de rendre compte de l'énoncé du Menteur et de stopper son auto-engendrement. Cependant, une autre approche de résolution da paradoxe du Menteur a aussi été préconisée : elle consiste à rechercher, à lintérieur même du langage naturel, les moyens de résoudre le paradoxe.

II.2. Résolution du paradoxe du menteur en langage naturel : La théorie des Catégories Sémantiques de RL. Martin et de L. Goldustein en logique trivalente.

R.L. Martin et L. Goldstein ont élabore la Théorie des Catégories Sêmantiques đont lìdée de base est qu'un sujet ou un prédicá, une fois inséxés dans un énoncé, imposent des restrictions sémantiques aux expressions qui formeront leur contexte strict.

La volonté de construire une thécrie à partir des informations que nous livre Ie langage naturel est due au fait que R.L. Martin et L. Goldstein estiment que le recours à mn langage hiérarchisé fornel sanctionne trop de phrases sui-référentielles ơn langage naturel. Nous avons, en effet, pu 
constater que le langage hiérarchisế de B. Russeli (il en est de même pour celui de A. Tarski ) exciut toutes les płrases sui-référentielles sur la base de leur malformation syntaxique. L'abandon de toutes les phrases suiréférentielles du langage naturel est un prix trop coûteux pour R.L. Martin et L. Goldstein ; aussi leur but sera de pouvoir séparer, grâce à la Théorie des Catégories Séranantiques, la "bonne" de la "maurvaise" sui-référence.

Le fondement de la Theorie des Catégories Sérantiques est ainsi đéfini par L. Goldstein : "la notion de faute de catégorie est fondée sur lidée intuitivement claire qu'il y a des catégories d'expression, telles que le résultat d'unir n'importe quel menbre d'une catégorie avec n'importe quel membre óune autre catégorie, est ane phrase grammaticalement correcte qui ne peut être utilisée pour dire quoi que ce soit de vzai ou de faux". (1981, p. 405).

Une phrase qui contient une faute de catégorie est donc constituée o'un sujet et d'un prédicat appartenant à deux catégories sémantiques distinctes, ce qui a pour conséquence que Ia catégorie du sujet est incompatible sếmantiquement avec celle du prédicat (on inversement). Seules les phrases sémantiquement correctes pourront être évaluxées en termes de "vrai" ou "faux", les phrases contenant une faute de catégorie étant "ni vraies ni fausses". R.L. Martin et L. Goldstein vont donc élaborer des tests sémantiques qui permettront de déterminer les diverses catégories sémautiques qai peuvent être contenues dans une phrase. L'élaboration des catégories sémantìques a donc pour but de doter de concepts opératoires une théorie linguistique qui travaille à lintérieur du langage naturel. Les phrases qui ne pourront passer avec succès les tests sémantiques de R.L. Martin et de L. Goldstein contiendront une faute đe catégorie sémantique. L'incorrection sémantique décelée permettra de comprendre pourquoi ces phrases n'ont pas de signification. Les phrases contenant une faute de catégorie ne seront donc jamais considérées comme susceptibles de pouvoir exprimer une proposition. 
La procédure permettant la détermination de la correction sémantique d'une phrase est constituée de quatre étapes qui délimitent les conditions devant être remplies par une phrase pour qu'elle ait une valeur de vérité. Ainsi :

A. La première étape pour déterminer si une phrase donnée est sếmantiquement correcten, est de déterminer le domaine d'applicabilitié du prédicat de la phrase.

Il s'agit donc de déterminer lensemble des individus qui sont dans l'extension du prédicat de la phrase.

La définition suivante peut donc être posée : $(1970$ \& 78, p. 92)

Une phrase de la forme $\mathrm{Fa}$ est sémontiquement correcte si et seulement si $a \in R A(F)$; elle est sémantiquement incorrecte si et seulement si a $\notin R A(F) .1$

B. La seconde étape de notre procédure est de déterminer si la phrase est ou n'est pas sui-référentielle.

C. La troisième étape de notre procédure s'applique senlement aux phrases non-sui-référentielles. Blle nous dit de déterminer si le sujet dune phrase indique ou n'indique pas, sur la base de son sens, la sorte de chose incluse dans le domaine d'applicabilité du prédicat ; d'appeler la phrase "sémantiquement correcte" si et seulement ceci est.

D. La quatrième étape de notre procédure conceme senlement les phrases-types sui-référentielles. Nous sommes chargés, pour toutes les phrases-types sui-référentielles, de déterminer si la référence démonstrative diu sujet est incluse dans le domaine d"applicabilité du prédicat, et d'appeler la phrase "sémantiquement correcte" si et seulement si elle l'est (1967, pp. 292-294).

1 RA signifie "rang dapplicabilité", soit "domaine d'applicabilité". 
Une expression sujèt peut avoir une référence démonstrative et une référence propre. Ainsi, si l'expression est utilisée avec succès pour sélectionner un objet, alors cet objet est la reférence démonstrative de lexptession Par contre si lobjet est conforme à la description qu'en a donné l'expression, alors l'objet est la référence propre de l'expression. Une expression aura donc à la fois une référence démonstrative et une référence propre, si elle permet d'identifier et de décrixe l'objet. Le choix de tester la correction sémantique d'une phrase sui-référentielle sur sa référence démonstrative plutôt que sur sa téférence propre se justifie par le fait que l'echec de référence propre est accepté par R.L. Martio.

Nous allons maintenant appliquer cette procédure au paradoxe du Menteur :

Le paradoxe du Menteur simple considéré en tant que type, car toutes ses occurrences sous forme de token sont suj-réfétentielles, sera réécrit par R.L. Martin, de la façon suivante :

(a) Cette phrase (sémantiquement correcte) est fausse.

car parler a'une phrase implique qu'on veut parler d'une phrase sémantiquement correcte.

L'étape $A$ de la procédure indique que nous devons déterminer le domaine d'applicabilité du prédicat "est farx". Etant donné que seules les phrases qui peuvent être évaluées en termes de "est vrai" ou "est faux" sont les phrases sémantiquement correctes, nous en déduisons que le domaine d'applicabilité du prédicat "est faux". est constitué de la classe des phrases sémantiquement correctes.

A l'étape $B$, nous constatons que (a) est une phrase sui-référentielle, puiscque son sujet mentionne la phrase elle-mêne qui est en usage. 
Nous devons donc directemient passer à l'étape $D$ pour déterminer si la référence démonstrative de son sujet est incluse dans Ie domaine d'applicabilité du prédicat : "est faux". Mais la référence démonstrative du sujet de (a) est (a) elle-même. Donc, avant de pouvoir répondre à la

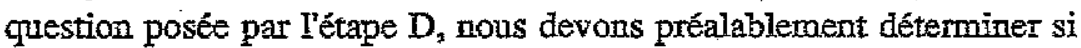
(a) est sémantiquement correcte. Nous sommes donc en présence d'un cercle vicieux, car (a) ne peut passer le test $D$ seulement après qu'elle ait passé ce test. La conclusion de R.L. Martin est la suivante (1967; pp. 299-300) : "D'accord, la procédure ne produit pas le résultat que (a) est sémantiquement incorrecte ; ce que nous voyons plutôt est que (a) ne peut jauais être raisonnablement déclarée sémantiquement correcte (...). Puisqu'on ne peut jamais dérnontrer que (a) est sémantiquement correcte, il n'est pas raisonnable d'appliquer la Ioi du tiers-excln à (a)". L'énoncé dur Menteur n'est done ni vrai ni faux.

La notion de référence démonstrative jone done un rôle très important lors de la résolution du paradoxe du Menteur, car elle permet de montrer qu'il y a un cercle vicieix qui empeche (a) de passer le test avec succès.

En conclusion, les trois résolutions qui viennent d'ètre exposées montrent que l'étude du paradoxe du Menteur consiste à xnettre à jour, d'une part, que Ie terme-sujet est en échec de référence (P.F. Strawson, B. Van Fraassen), et d'autre part, que l'attribution du prédicat de fausseté est inapplicable (R.L. Martin, L. Goldstein), ou mal appliquée (B. Russell). Par conséquent, ces trois résolutions ont toutes en commun de résoudre le paradoxe du Menteur à partir d'une analyse de ses constituants. La cause de l'engendrement du paradoxe est alors déterminée en fonction de cette analyse des constituants, et nous avons pu constater que ces théries linguistiques bivalentes et trivalentes convergent sur le fait que le paradoxe du Menteur est dû à une des trois causes suivantes : la présence d'une régression infinie, d'un cercle vicieux ou d'une confradiction à l'intérieur de la structure mêtne de cet énoncé. 
Cependant, expliquer la cause de l'engendrement du paradoxe dn Mienteur grâce à la mise en évìdence de la présence potentielle d'une régression infinie on d'un cercle vicieux ne nons semble pas une justification suffisamment approfondie. En effet, la régression infinie on le cercle vicieux ne sont pas, à notre avis, la cause de l'engendrement du paradoxe du Menteur, mais la caractérisation du raisonnement qu'il engendre. De plus, estimer que la cause de l'engendrement du paradore du Menteur est due à la présence đ'une contradiction à l'intérieur de sa structure interne est une explication erronée, car elle omet totalement la dimension temporelle de ce paradoxe. En effet, face au paradore du Menteur, nous n'obtenons jamais simultanément Cet énoncé est foux et cet énoncé est vrai, puisque linversion du contenu propositionnel de I'énoucé est le tésultat đur raisonnement engendré par le paradoxe. B. Van Fraassen avait bien vi ce problème, lorsqu'il accusait son formalisme d'être inadéquat à rendre compte des énoncés sui-falsificateurs : "Si X est, par exemple, la phrase du Menteur Ce que je dis maintenant est faux, rous voyons que son déni tiest pas exprimé par Ce que je dis maintenant $n^{\prime}$ est pas faux. Si le Menteur devait énoncer les deux, il aurait à les énoncer suxcessivement, aussi le maintenant téférait à deux temps différents. Donc, il the dénierait pas son premier énoncé en prononçant le deuxième, le deuxième référant senlement à lui-mêtme" (1968, p. 147).

Aussi nous nous proposons de montrer que la cause de l'engendrement du paracoxe du Menteur ne peut pas être décelée grâce à une analyse préalable đes termes constitatifs de cet énoncé. Nous mettrons ainsi en évidence que le paradoxe du Menteur doit être appréhendé en tant qu'énoncé (dans toute sa totalité) avec pour spécifité de donner lien à l'élaboration d'un raisonnement de nature leibnizienne.

De plus, l'approche préconisée par les trois résolutions précédemment exposées nous semble sujette à caution pour les deux raisons suivantes : 
- Cette approche est toujours externe : en effet, une élaboration préalable de la thérie est effectuée et cette théorie est easuite appliquée an paradoxe du Menteur.

- Cette approche se présente toujours de façon statique : on applique la théorie, on bloque l'engendrement du parađoxe en mettant en évidence sa canse et on évahre sa valeur de vérité. Le dynamisme interne caractéristique du paradoxe du Menteur n'est donc jamais pris en compte dans cette approche.

Dans ces theories, le paradoxe du Menteur, après résolution, n'en reste pas moins un énoncé pathologique, puisque B. Van Fraassen le considère comme un cas limite, B. Russell et R.L. Martin comme un énoncé syntaxiquement et sémaníquement mal formé.

Nous nous proposons maintenant, après avoir dégagé les idées essentielles communes aux thêonies linguistiques bivalentes et trivalentes, de présenter une approche de résolution dynamique du paradoxe du Menteur, c'est-à-dire une approche qui sera plus conforme à la nature du problème à résoudre. Cette approche consistera à laisser librement évoluer l'engendrement cu paradoxe, de telle façon qu'il deviendra alors possible de suivre son processus d'évahuation et de calculer son instabilité sểmantique. Il s'ensait que cette approche sera de plus inteme.

\section{Résolution dynamique du paradoxe du Menteur}

La première résolution du paradoxe du Menteur dans le cadre d'une approche dynamique a été proposée par H. Herzberger en 1982. Cette approche de résolution dynamique du paradoxe est appelée Sémantique

Nã̃ve car elle se définit par le fait qu'elle encourage l'engendrement du paradoxe du Menteur et qu'elle ne tire de conclusions théoriques que dans le cas où un équilibre émergerait de son processus d'évaluation. Ainsi, en aucurn cas le paradoxe du Menteur ne sera bloqué et tésolu grâce à la prise en considération qu'il renferme une contradiction interne ou qu'il 
contient un cercle vicieux on une régression infinie. L'approche diz paradoxe du Menteur dans le cadre de la Sémantique Nä̈ve est done la suivante : "Plutôt que d'essayer d'éliminer ces paradoxes, je veux consìdérer l'expérience de les encourager positivement à survenir et à les regarder trouver leur propre issue. Cette approche, que j'appelle la sémantique naũve, est un exercice délibérément non-directif. L'idée est de se tenir à l'écart et de laisser les paradoxes révéler leurs principes internes" (H. Herzberger, 1982).

Anzssi, l'observation de l'évolution du Paradoxe du Menteur sera effectuée:

- en logique binaire, puisque cet énoncé révèle son instabilité lorsqu'il est évalué dans une logique classique. L'introduction d'une logique nonclassique anrait d'ailleurs pour conséquence d'orienter l'évolution du paradoxe, or aucune restriction ne doit être placée qui ne soit dictée par le paradoxe du Menteur lui-mêtme.

- dans tne conception hiérarchisée du langage naturel, de façon à ce qu'il devienae possible dinscrire l'évolution dynamique du paradoxe et de suivre niveari par niveau son instabilité sémantique (Cet énoncé est faux à la condition quil soit vrai). Aussi, malgré les critiques que nous avons adressées à l'approche des théories statiques, nous conserverons cependant les méthodes qu'elles ont préconisées, puisque notre étude du paradoxe du Menteur s'effectuera au sein d'nn langage naturel hiérarchisé. De même, nous n'excluons pas la possibilité d’avoir recours, comme R.L. Martin et L. Goldstein, à des concepts opératoires ; à la différence près que nos concepts ne seront jamais préalablement construits, mais deduits des informations liviées par le paradoxe luimême.

Cependant, si le cadre théorique de la Sémantique Narve ci-dessus définit par $\mathrm{H}$. Herzberger nous paraît digne d'intérêt car le dynamisme interne da paradoxe du Menteur y est privilégié, nous avons ez toutefois 
loccasion de montrer ${ }^{1}$ que la résolution proposée par H. Hexzberger du paradoxe du Menteur est sujette à caution, car elle ne respecte pas suffisamment l'evoletion naturelle du paradoxe. Aussi, nous nous proposons de présenter, tout en restant dans la perspective de la Sémantique Naïve, une gouvelle approche de résolution quai aura pour fondement un respect de l'évolution naturelle du parađoxe.

Dans ce but, nous allons tout d'abord commencer notre étude du paradoxe du Menteur en mettant en évidence la cause de son engendrement. Puis, nous présenterons l'approche de résolution dynamique que nous proposons et la résolution du paradoxe du Menteur sera exposée. Celle-ci nous permettra alors de mettre à jour que f'instabilité sémantique du paradoxe du Menteur est due à mo sousdétermination đe son contenu propositionnel.

\section{III.1. Cause de l'engendrement du paradoxe du Menteur}

Nous nous proposons de déterminer la cause de l'engendrement du paradoxe du Menteur dans le cadre de la Semantique Naïve, c'est-à-dire en fonction de son seul contenu propositionnel.

Ainsi, le raisonnement intuitif qui peut être élaboré en fonction du seul contenu propositionnel du paradoxe du Mentelir sous sa forme simple, et qui carrespond à la première phase de son engendrement, est le suivant:

A: "Cet énoná est faux".

(1) Si cet énoncé est faux, alors il est vrai que cet énoncé est faux.

(2) Mais cet énoncé indiquait de Iui-même qu'il était faux.

(3) Donc il disait la vérité ; et par conséquent : cet énoncé est vrai.

${ }^{1}$ Cf. GODAKT, B., 1987. 
Maintenant que le paradoxe nous a révélé, à partir đe son seul contenu propositionnel, son principe interne d'engendrement, nous avons la possibilitế de retranscrite ce raisonnement intuitif de façon pius formelle, afin d'en déceler la cause.

Nous pouvons tout d'abord réécrire l'énoncé A, sous la forme:

$A=$ Fanx (Cet Énoncé ) ; soit: $A=F(C e t$ énonce) $)$

Puis nous pouvons constater que l'étape (1) consiste simplement à poser que, puisque lénoncé $A$ a êté prononcé, A s'est présenté de luimềme comme étanì vrai. Le raisonnement intuitif ssest donc élaboré en fonction de cette présomption de vérité.

Ainsi, plus formellement :

(1) estéquivalent à : si $\mathrm{A}$, alors $\mathrm{V}(\mathrm{A})$

et il s'ensuit que (1) pemet de déduire V(A); c'est-à-dire, si nous remplaçons A par sa valeur:

(1) permet de déduire : VIF (Cet énoncé $))$.

L'étape (2) restitue la référence sous-jacente à (Cet énoncé) dans $F$ (Cet énoncé) de l'étape (1), grâce à la prise en considération de l'autoréférence ("indiquait d'elle-même"). Ceci signifie que la référence de (Cet énoncế) va être sanurée grâce à la substitution :

Cet énoncé $=\mathrm{F}$ (Cet énoncé) 
et par conséquent, l'étape (2) permet de déduire, en fonction de $F$ (Cet énoncé) de l'étape (1), l'équivalence suivante :

$F($ Cet énoncé ) est identigue à $F(F($ Cet énoncé $))$.

La présence de limparfait, dans l'śtape (3), permet de considérer que (3) référe au contenu proposśtionnel de l'étape (1). La fonction de l'étape (3) consiste donc à vérifier la presomption de vérité qui a été établie à l'étape (1) grâce à la prise en considération de l'équivalence obtenue à l'étape (2). Ainsi, l'étape (3) va vérifier que l'énoncé đa Menteur est vrai, en effectuant une substitution du membre de droite $\mathrm{F}(\mathrm{F}$ (Cet énoncé)) au membre de gauche $\mathrm{F}$ (Cet énoncé) de l'équivalence, dans $\mathrm{V}$ ( $\mathrm{F}$ (Cet énoncé)) de l'étape (1). Soit :

$\mathrm{V}(\mathrm{F}(\mathrm{F}(\mathrm{Cet}$ enoncé) $))$; donc :

$V(V(C e t$ énoncé) $)$.

En rêsumé :

- L'étape (1) pose que A est vrai.

- L'étape (2) restitue la référence de A par substitution, et il s'ensuit qu'une équivalence entre deux énoncés est obtenue.

- L'étape (3) effectue une substitution du membre de droite de I'équivalence dans (1), et pose que $\sim$ A est vrai, soit que : A est faux.

Il s'ensuit que cette première analyse permet de considérer que le contenu propositionnel d'un énoncé sui-falsificateur engendre de luimême un raisonnement de la forme d'une substitution leibnizienne qui ne respecte pas la loi de Leibniz. En effet, nous avons un énoncé A tenu pour vrai et une équivalence entre deux énoncés qui ont pour consécquence qu'une substitution leibnizienne est réalisête, tout en êtant violée.

En fonction de cette caractérisation de l'engendrement du paradoxe du Menteur, nous pouvons maintenant tenter de rechercher sa cause. Ainsi, nous pouvons tout d'abord remarquer que le raisommement qui est 
engendré par le paradoxe est une forme élaborée de substitution leibnizienne, puisqu'il repose sur deux substitutions successives. En effet, l'équivalence posée à l'étape (2) entre les deux énoncés a été obtenue grâce à une première substitution, d'un énoncé à une expression, et cette Équivalence a donné lien à la rélisation d'une seconde substitution, qui,

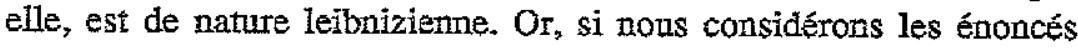
auxquels on applique habituellement la loi de Leibniz, nous pournons nous renđ̈re compte que l'équivalence posée entre les deux expressions identiques n'est jamais obtenue grâce à une première substitution, comme dans le cas du paradoxe du Menteur, mais grâce à tro connaissance extérieure que possède le locuteur. En effet, les cas les plus fréquents sont du type :

(1) Jean travaille beancoup ces temps-ci.

(2) Jean est identique à Monsieur Dupond ; d'où :

(3) Monsieur Dupond travaille beancoup ces temps-ci.

Nous pouvons en effet considérer que l'équivalence posée en (2) n'est pas due à une substitution, mais au fait que le locuteur sait que Jean s'appelle Monsieur Dupond. La preuve en est que si le locutenr n'avait pas possédé cette connaissance extérieure :

\section{Jean est identique à Monsieur Dupond}

Yean, pour des raisons quelconques, n'ayant jamais eu l'occasion de lui dire son nom de famille; alors, ce même locuteur eût été incapable d'effectuer la substitution leibnizienne de l'étape (3).

Dans le paradoxe du Menteur, l'ếquivalence obtenue à l'étape (2) est le résultat d'une première substitution, de par la présence de lautoréférence qui rend caduque tout appel à une connaissance extérieure. Or, si nous nous intéressons plus précisément à l'opération 
effectué par cette première substitution, nous pouvons constater que cette substitution s'opère entre une expression (Cet énoncé) et un énoncé $F$ (Cet énoncé) et qu'elle a pour conséquence que l'śquivalence suivante va pouvoir être posée :

$\mathrm{F}($ Cet énoncé) est identique à $\mathrm{F}(\mathrm{F}(\mathrm{Cet}$ éroncé) ) ; soit :

"Cet énoncé est fanx" est identique à "Cet énoncé est vaai".

Il s'ensuit que cette première substitution a pour effet de poser une équivalence entre deux énoncés opposés. Or, une substitution leibnizienne doit toujours s'opérer en fonction deux énoncés identiques. Il n'est done pas surprenant que la deuxième substitution qui s'opère à l'étape (3) en fonction de cette équivalence, ne respecte pas la loi de Leibniz

$\mathrm{Par}$ conséquent, la cause de l'engendrement du paradoxe suifalsificateur se situe dans la première substitution qui a opérée la saturation de la référence de (Cet énoncé). Plus précisément, la décomposition, présentée ci-dessus, de l'operation effectuée par cette première substization nous permet de considérer que l'engendrement du paradoxe đu Menteur est đû à l'enchânement successif de deux causes :

La première cause est l'autoréférence qui a pour conséquence que la substitution va être effectuée en fonction d'une faute de catégorie sémantique. En effet, la substitution s'est opérée entre une expression "Cet énoncé" et un énoncé: "Cet énoncé est faux"; alors qu"elle aurait dût s'effectuer entre deux éléments linguistiques de même nature.

Cependant, l'autoréférence n'est pas à elle seule une cause suffisante pour qu'il y ait engendrement du paradoxe. En effet, si nous considérons l'énoncé autoréférent suivant:

"Cet énoncé est vrai" 
la substitution qui opèrera la saturation de la refférence da sujet "Cet énoncé" s'effectuera, comme dans le cas de l'énoncé du Menteur, en fonction d'une faute de catégorie sémantique:

Cet énoncé $=$ Cet énoncé est vrai.

Pourtant, l'application d'une substitution leibnizienne à cet ênoncé n'aura pas pour conséquence un non-respect de la loi de Leibniz, mais l'apparition d'une simple redondance. En effet :

(1) Cet ếnoncé est vrai ; soit $V$ (Cet énoncé)

(2) $V($ Cet énoncé) est identique à $V(V(C e t$ énoncế)); par saturation, grâce à la substitution, de la référence de (Cet énoncé).

(3) D'où, substitution leibnizienne du membre de droite de l'équivalence posée en (2), dans (1): $\mathrm{V}(\mathrm{V}($ Cet énoncé) ) ; soit $V($ Cet énoncé).

Par conséquent, l'engendrement du paradoxe du Menteur est dô à la présence d'une seconde cause : les deux prédicats de fausseté, mis en présence par l'autoréférence à l'́́tape (2) du raisonnement, ont interagi et ont eu pour résultat d'inverser le sens du contenu propositionnel de l'énoncé du Menteur. En effet, nous pouvons considérer que l'équivalence posée, grâce à la première substitntion, est une équivalence entre deux énoncés opposés, car dans le membre de droite de l'équivalence, les prédicats de fausseté ont interagi et ont eu pour conséquence que le sens du contenu propositionnel de l'énoncé du Menteur a été inversé.

Par conséquent, nous pouvons ainsi résumer le rôle successif jouné par les deux causes de l'engendrement du paradoxe du Menteur : l'autoréférence prépare la structure paradozale en permettant qu'une équivalence puisse être posée et en rendant possible la mise en présence 
simultanée des deux prédicats de fausseté. L'interaction sémantique des deux prédicats de fausseté qui en résulte, a pour conséquence que l'équivalence s'établira en fonction de derx énoncếs opposés.

La spécificité de lengendrement du parádore da Menteur peut donc encore être précisée : un énoncé sui-falsificateur se caractérise par le fait d'engerdrer un raisonnement de La forme d'ume substitution leibnizienne, qui ne respecte pas la loi de Leibniz, car la substitution s'opère en fonction d'une équivalence établie entre deux énoncés opposés.

Aussi, puisque nous savons maintenant que le dynamisme interne du paradoxe cu Menteur peut être nettement décelé grâce au non-respect de la loi de Leibniz qu'il occasionne, nous nous proposons, lors de la résolution que nous allons présenter, d'effectuer systématiquement des substitations leibniziennes, afin de nous assurer que le paradoxe a ́́té effectivement résolus.

Nous allons maintenant présenter l'approche que nous proposons pour une résolution du paradoxe du Menteur sous sa forme autoréférente directe.

\section{III.2. Approche proposée : explication de la procédure}

De façon à respecter l'approche théorique de la Semantique Naïve, nous allons présenter une analyse intuitive du processus d'évaluation our paradoxe du Menteur sous la forme:

Socrate : Socrate dit faux

Robert: Socrate dit vrai

Cette formulation du paradoxe du Menteur est la version de Buridaz, et rous montrerons qu'elle se rêduit à celle du paradoxe autoréférent direct. 
Nous allons donc tout d'abord laisser le paradoze révéler ses principes internes, puis nous montrerons que cette première étude permet de constituer un langage hiérarchisé où l'évolution đynanique du parađoxe peut s'inscrire. Ainsi :

- Le premier principe inteme qui peut être mis en évidence en fonction du contenu propositionnel du paradoxe du Menteur, concerne l'attribution respective des valeurs de vérité de ces deux énoncés.

En effet, lorsque l'énoncé đe Socrate est commenté par Robert 1 , nous pouvons constater que l'énoncé de Robert confirme systématiquement l'énoncé de Socrate, puisque Robert dit Socrate dit vrai. Aussi, nous pouvons en déduire que l'énoncé de Robert et l'énoncé de Socrate doivent avoir les mêmes valeurs de véritế. Mais, puisque l'énoncé de Socrate est la négation de celui de Robert, ces dewx énoncés doivent avoir des valeurs de vérité opposées. Nous obtenons donc le cercle vicieux suivant quant à l'attribution respective des valeurs de vérité de ces deux énoncés :

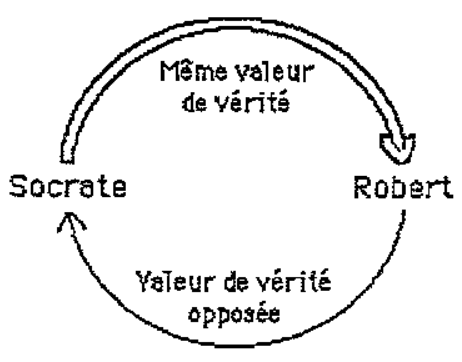

- Le second principe interne au paradoxe peut être obtent en tenant compte de la natare prédicative de "est vrai". Ainsi, si "est vrai" est analysé comme un prédicat, alors, étant donné que l'énoncé de Socrate est

1 Ceci explique le sens de la flèche dans le schéma. 
jugé "vrai" par l'énoncé de Robert (cf. la note précédente), l'énoncé de Robert doit se sitner au niveau inonédiatement supérienr à celui de Socrate. Toutefois l'énoncé de Socrate étant la négation de celui de Robert, ces coux énoncés doivent aussi se situer sur le même nivean de langage. Nous obtenons donc un second cercle vicieux quant aux règles de passage dans la hiérarchie du langage:

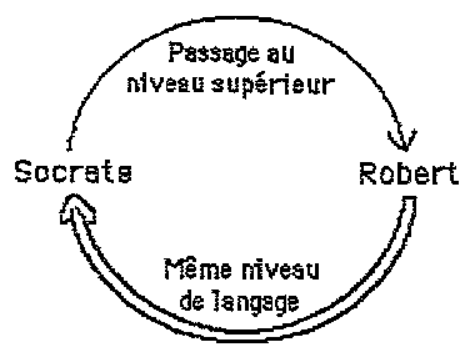

Etant donné que nous voulons conserver la dynamique interne du paradoxe du Menteur, les deux cercles vicieux qui viennent d'être obtenus ne seront pas bloqués en mettant en évidence çu'ils peuvent être réduits à đes contradictions, mais ils seront pensés comme des processus sérantiques en interaction à travers lesquels les valeurs de vérité des deux énoncés penvent être déterminées pour les deux premiers niveanx les plus inférieurs du langage hiêrarchisé (soit, ici : $n$ et $n+1$ ). Les deug cexcles vicieux s'engend́rent, respectant ainsi la dynamique inteme du paradoxe. Par consequent, la façon de construize Ie langage hiérarchisé est déterminé par le paradoxe hri-même, de telle façon que son évolution dynamique puisse y être inscrite. L'approche de résolution que nous allons proposée est donc bien interne. 
Nous obtenons donc en uritisant les deux priacipes internes ci-dessus 1 :

Socrate

Robert

n Socrate dit faux

Socrate dit vrai

$n+1 \quad F(V($ Socrate dit faux))

Socrate dit wrai

$\mathrm{V}$ (Socrate dit faux)

Socrate dit farzx

La procédure de résolution que nous proposons sera donc la suivante :

- Au niveau n, le locuteur écoute les énoncés de Socrate et de Robert sans leur attribuer de valeur de vérité, et détermine les règles de passage au nivean $n+1$ en fonction du contenu propositionnel des deux énoncés quili vient d'entendre. Par conséquent, au nivean n, Ie locutenr observe les univers de croyance de Socrate et de Robert, sans prendre position, car il doit d'abord laisser le paradoxe s'établir (cf. le tableau ci-dessus).

- An ziveau $n+1$, le locuteur est incapable de se prononcer sur la valeur de vérité de ces deux énoncés et il en sera de même au niveau supérieur $n+2$. Etant dorné que ce paradoxe pose le problème sémantique et logique de sọn évaluation, le locuteur est condrzit à choisir un système intexprétatif qu'il détermine en fonction de la nature mềme du. problème qu'il a à résondre. Par conséquent, le locureur doit envisager au nivean $n+1$, les quatre possibilités sémantico-logiques suivantes :

- Socrate et Robert disent tous les deux vrai.

- Socrate et Robert disent tous les deux fanx.

- Socrate dit vrai et Robert dit faux.

- Socrate dit faux et Robert dit vrai.

1 Le double trait permet de marquer que Ia valeur de vérité de l'énonce de Socrate est conservé, puisque Robert confirme cet énoncé. Le trait simple signifie que la valenir de vérité de l'énoncé de Robert est inversée, puisque Socrate infirme ce que dit Robert. 
C'est au moment où le locuteur va effectuer ces quatre cas de figure que les énoncés fe Socrate et de Robert vont être subordomnés à l'univers de croyance du locuteur. Les substitutions leibniziennes qui auront lieu dans tous les niveaux supérieurs s'effectueront à l'intérieur de l'univers de croyance du locuteur, et elles prendront en compte l'énoncé de Socrate et de Robert, teil qu'il est considéré par le locuteur au niveau strictement inférieur, afin de respecter la cohérence de son univers de croyance. Les substitutions leibniziennes seront donc opérées de façon inductive, suivant la loi :

$$
\mathrm{S}_{\mathrm{n}, \mathrm{n}+1}, \mathrm{~S}_{\mathrm{n}+1, \mathrm{n}+2} \ldots
$$

De plus, les règles de passage concemant l'attribution de la valeur de véritse des dewx énoncés seront établies đe niveau en niveau, en fonction di contenu propositionnel des énoncếs qui viendra d'être obtenu. Les règles de passage de la hiérarchie du langage restent valides pour tous les niveaux supẹrieurs à $n+1$, car seul Robert, en attribuant une valeur de vérité à l'énoncé de Socrate, permet un passage au niveau supérieur.

Quand nous avons effectué les qratre schémas correspondant aux quatre univers de croyance que le locuteur peut envisager lorsqu'il entend les énoncés de Socrate et de Robert, nous avons constaté que les deux premières possibilités sémantico-logiques présentent une structure très instable 1 et que seuls les cas où "Socrate dit vrai et Robert dit faux", et "Socrate dit faux et Robert dit vrai" peuvent être stabilisés. Nous allons par conséquent n'exposer que ces deux derniers cas. 1 II n'y a plus précisément aucun point de convergence et il s'ensuit que le paradoxe
ne se stabitise pas, même momentanérnèt. 
- Cas où dans l'univers de croyance du locuteur, Socrate dit vrai et Robert dit faux :

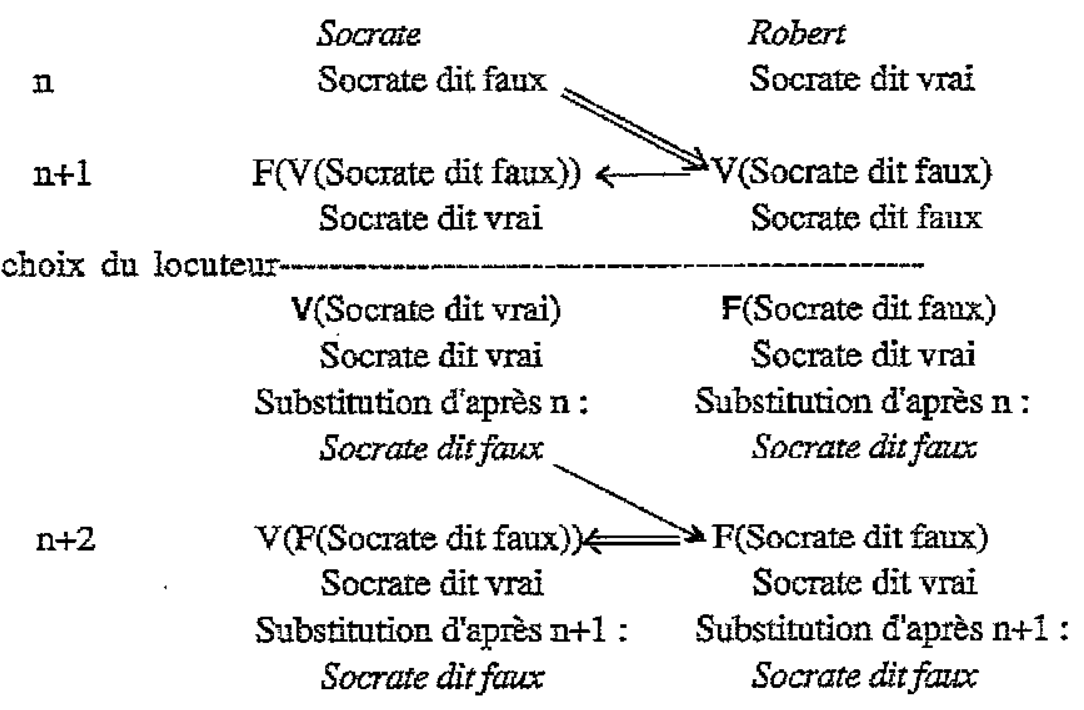

La procédure se repète puisque les énoncés obtenus aur niveau n+2 sont identiques alix énoncés du nivean $\mathrm{n}+1$.

Par consếquent, nous pouvons considérer que le paradoxe se stabilise dès le niveau $n+1$, puisque Socrate et Robert s'accordent sur le fait que "Socrate dit foux". Il s'ensuit que la détermination de la valeur de vérité de l'énoncé de Socrate devient stable sur la valeur "faux", et que cet énoncé cesse d'être un paradoxe.

Cependant une objection pourrait être faite à cette résolution, qui consisterait à dire que les hypothèses qui ont été faites cans l'univers de croyance du locuteur Socrate dit vrai et Robert ment, avaient déjà stabilisé le paradoxe, puisqu'elles ont pour conséquence que Socrate et Robert s'accordent sur le fait que "Socrate dit vrai". 
La réponse à cette objection est que la "stabilisation", due à la présence des hypothèses, ne peut pas être considérée comme une véritable stabilisation, car nous savons que seule l'application d'une substitution leibnizienne, en fonction du contenu propositionnel de l'énoncé du Menteur, peut nous assurer que Ie paradoxe est effectivement stabilisé. En effet, ce rest pas parce qu'on fait l'hypothèse que "Socrate dit rrai" que cet ênoncế, une fois substitué à "Socrate dit faux", empêchera Ie paradoxe de se réengendrer (cf. le raisonnement intuitif, exposé en III.1, où la même hypothèse a été faite à l'étape (1) et où le paradoze s'est réengendré : étape (3)). $\mathrm{Or}$, au niveau $\mathrm{n}+1$, après que les hypothèses aient été posées, nous avons effectivement réinjecté le paradoxe du Mentenr "Socrate dit faux", puisque nous avons opéré la substitution leibnizienne en forction du niveaun $n$. Par consequent, le paradoxe aurait très bien pu se réengendrer.

Nous allons maintenant examiner la seconde résolution concurrente qui peut être faite du paradoxe du Menteur sous sa forme simple :

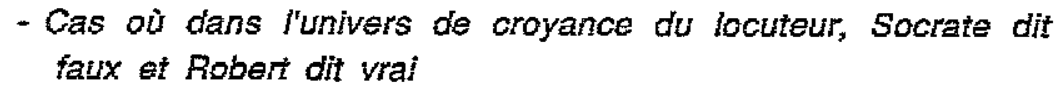

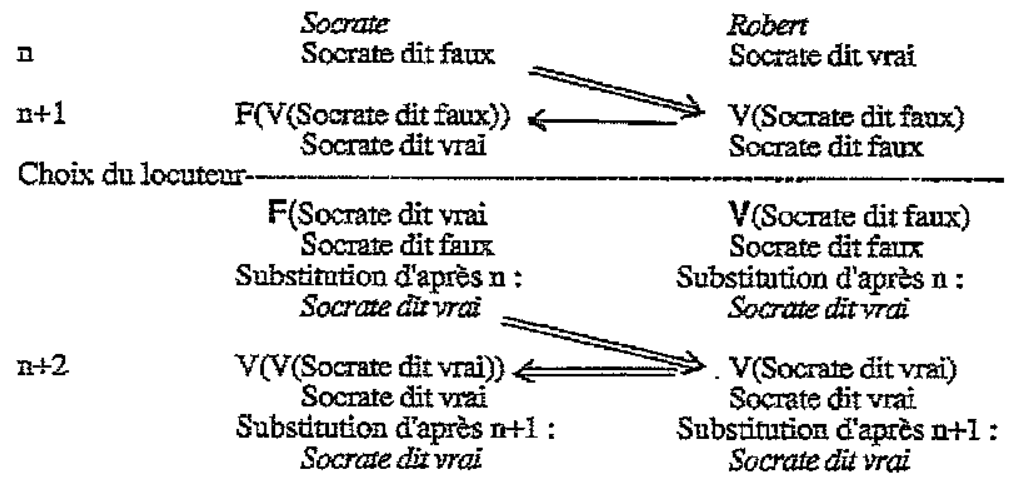


A partir du niveau $\mathrm{n}+2$, la procédure se répétera, car les énoncés obtenus au niveau $n+2$ sont identiques aux énoncés du niveau $n+1$.

II s'ensuit que le paradoxe se stabilise dès le niveau $n+I$ sur la valeur vrai, puisque Socrate et Robert s'accordent sur le fait que Socrate dit vrai. L'énoncé du Menteur cesse donc d'être un paradoxe.

Nous tenons ici à faire remarquer que, comme dans le cas précédemment étudié, tes hypothèses faites par le locuteur, dans son univers de croyance, ont pour conséquence que Robert et Socrate disent tous les deux : Socrate dir fautx. Cependant, étant donné que lors de la substitution leibnizienne qui esi immédiatement effectuée, le paradoxe đu Menteur est réinjectế, puisque l'on substiłue à Socrate dit faux, l'énoncé dui Menteur don niveau $n$, il était tout-à-fait envisageable que le paradoxe du Menteur se réengendre. Or, puisque cette substitution leibnizienne, en fonction du paradoxe đu Menteur lui-même, n'a pas eu pour consséquence un non respect de la loi de Leibniz, nous pouvons en déduire que le paradoxe est effectivement resoln.

Toutefois, il pourrait nous être objecté que les deux résolutions, que nous venons de présenter, ne sont valables que pour la version de Buridan du paradoxe du Menteur:

Socrate: "Socrate dit faux".

Robert: "Socrate dit vrai".

mais qu'elles ne permettent pas de rendre compte du paradoxe du Menteur ainsi exprimé :

Socrate: "Socrate dit faux".

Cependant, si nous considérons l'énoncé de Robert, nous pouvons d'une part, constater que celui-ci n'altère en rien le contenu propositionnel de l'énoncé du Menteur, puisqu'il ne fait que de le 
confinmer. Par conséquent, l'énoncé đe Robert a pour fonction d'exprimer la présomption de vérité que nous avions pu déceler à l'étape (I) du raisonnement intuitif obtenu en fonction du seul contenu propositionnel de l'énoncé du Menteur. D'autre part, si nous considérons les deux schémas de résolution précédemment présentés, nous pourvons constater que l'énoncé de Robert du niveau $\mathrm{n}, n^{\prime}$ est jamais pris en compte aux niveaux supérieurs, car dès le niveau $\mathrm{n}+1$, nous nous sommes toxjours référée à l'énoncé đe Socrate, donc à l'énoncé du Menteur. II s'ensuit que la formulation du paradoxe da Menteur de Buridar est équivalente au paradoxe cu Menteur sous Ia forme "Je mens".

Aussi, si nous avons choisi la version du paradoxe dn Menteur de Buridan, c'est parce qu'elle présente lavantage de rendre beaucoup plus explicite la résolution du paradoxe. En effet, cette version nous offre la possibilité de rendre compte du dynamisme inteme au paradoxe du Menteur, car en mettant en Évidence, grâce à l'énoncé de Robert, la présomption de vérité présente dans l'énoncé de Socrate, elle permet de générer le langage ziérarchisé. II s'ensuit que les résolutions du paradoxé que nous ayons proposées ont pu être suivies niveau par nivean, et que nous avons pu déteminer précisément le niveau où le paradoxe se stabilise definitivement.

De plus, si nous considérons les deux résolutions que nous avons obtenues, nous pouvons nous rendre compte que nous sommes face à un dilemme. En effet, si le locuteur tient pour vrai que Robert est urr mentelir et que Socrate dit la vérité, l'énoncé du Menteur aura la valeur de vérité "faux" dans son univers de croyance. Par contre, si le locuteur fait les hypothèses inverses (Socrate est un menteur et Robert dit la vérité), l'énoncé đu Menteur sera "vrai" dans son mivers de croyance. Par consequent, nous sommes en présence de deux raisonnements différents, te par le choix des hypothèses, qui donnent lien à deux conclusions opposées et équiprobables. Aussi, nous voudrions montrer gue le choix de la version du paradoxe du Mentezr de Buridan se justifie 
également par le fait qu'elle peut nous permettre de sortir de ce dilemme. En effet, notre but est de proposer une résolution du paradoxe suifalsificateur qui ne contrarie pas son dynamisme inteme. Or la version du paradoxe du Menteur de Buridan, parce qu'elle rend coropte explicitement de l'évolution dynamique du paradoxe sur les différents niveaux de langage, va nous permettre de contrôler si, dans les deux résolutions qui ont été exposés, le dynamisme propre au paradoxe du Menteur a bien été respecté.

Ainsi, la version du paradoxe du Menteur de Buridan, parce qu'elle met en évidence, grâce à l'énoncé de Robert, la présomption de vérité interne à l'énoncé de Socrate, nous offre la possibilité de suivre précisément, grâce au discours de Robert, les différentes étapes du raisonnernent intuitif que nous avions obtenues en fonction du seul contenz propositionnel de l'énoncé di Menteur. En effet, lorscue nous avons retranscrit formellement ce raisonnement intuitif, nous avons pu considérer que l'engendrement du paradoxe du Menteur s'effectuait en fonction de la présomption de vérité présente à l'étape (1) puisque l'engendrement du paradoxe du Menteur nous a été révélé à l'étape (3), lorsque nous avons constaté que cette présomption de vérité était infirmée. Par conséquent, étant donnế que l'énoncé đe Robert du niveau n exprime cette présomption de vérité, le discours de Robert au niveaur $n+1$ permet de reflêter l'évolution naturelle đu paradoxe. En effet, si nous effectuons le schéma qui représente le raisonnement de Robert, sans faire intervenir les hypothèses faites par le locuteur, nous obtiendrons : 
Socrate

Robert

n Socrate dit faux

Socrate dit vrai

$x+1$

V(Socrate dit faux)

Socrate dit faux

Substitution d'après n:

Socrate dit vrai.

et ce schéma correspond au raisomement suivant :

Robert: - Jaccorde au niveau n que : "Socrate dit vrai".

- Par consếquent : "Il est vrai que Socrate dit faux" an nivean $n+1$.

- Donc : "Socrate dit faux".

- Mais Socrate avait indiqué đe lui-même au niveau n qu'il disait faux. (Substitution daprès $n$ )

- Done : "Socrate dit vrais".

II s'ensuit que si nous laissons parler Robert, sans faire lnypothèse qu'il est un menteur ou qu'il dit la vérité, nous pouvons effectivement constater que le discours de Robert correspond aux différentes étapes du raisonnement intuitif que nous avions obtenues en fonction du seul contenu propositionnel de l'énoncé du Menteur. Par conséquent, le discours de Rober au niveau $n+1$ reflète exactement l'évolution naturelle du paradoxe. Aussi, étant donné que nous voulons proposer une résolution du paradoxe du Menteur qui respecte son dynamisme interne, nous allons maintenant exarziner les deux resolutions que nous avons présentées, afin de vérifier, grâce au discours de Robert, si l'évolution naturelle đu paradoxe a toujours été respectée. 
Ainsi, considérons tout d'abord le cas où, dans l'univers de croyance du locuteur, Socrate dit vrai et Robert dir faux. Nous savons que Robert, en exprimart au niveau $n$ une presomption de vérité, a indiqué qu'il tenait pour vrai l'énoncé de Socrate, et par conséquent qu'il était prêt à dire au niveau $\mathrm{n}+1$ : Il est wai que Socrate dit faux, donc Socrate dit faux. Or, 'lorsqu'au nivean $n+1$, le locuteur fair lhypothèse que Robert est m menteur, il contrarie l'évolution naturelle dus paradoxe, puisqu'il fait dire à Robert : Socrate dit vrai. PIus précisément, l'hypothèse faite par le locuteur empêche Robert d'avoir un discours cohérent, puisqu'elle ne lui permet pas de substituer à lintérieur de sa présomption de vérité i'énoncé de Socrate. Il s'ensuit que la première résohution qui a été présentée ne peut être considérée comme valide, puisqu'elle contrarie le dynamisme interne du paradoxe du Menteur.

Considếrons maintenant le cas où, dans l'univers de croyance du locuteur, Socrate ment et Robert dit la vérité. Nous ponvons immédiatement constater que lorsque le locuteur fait lhypothèse que Robert dit la vérité, il ne contrarie pas l'évolution naturelle đu paradoxe, puisqu'il fait dire à Robert : Socrate dit faux. Par conséquent, le locuteur respecte la cohérence du discours de Robert, en posant au niveau $n+1$ lénoncé qui coit être logiquenent déduit de la présomption de vérité énoncée par Robert au niveau n. Examinons maintenant si la seconde hypothèse faite par le locuteur: "Socrate ment" a pour conséquence d'altérer le dynamisme interne du paradoxe. Ainsi, nous pouvons constater que cette hypothèse a pour effet que Socrate ne dira pas an nivean n+1 : "Socrate dit vrai", mais "Socrate dit faux". Par conséquent, lorsque le locuteur ajoute cette hypothèse, il oblige Socrate à répéter son paradoxe. Or, étant donné que le locuteur va alors immédiatement effectuer une substitution leibnizienne en fonction du paradoxe du Menteur lni-même, il va offrir au paradoxe la possibilitê de se réengendrer. Ceci revient à dire qu'au niveau $n+1$, le locuteur a fait en sorte de réunir toutes les conditions qui peuvent permettre à un paradoxe 
d'exprimer son dynamisme interne. Par conséquent, la seconde résolution qui a été présentée est la seule valide, car elle stabilise définitivement le parađoxe sans contrarier son dynamisme inteme.

Cette étude du paradoxe du Menteur nous permet donc de mettre en śvidence que linstabilité sémantique de ce paradoxe, tel qu'il est habituellement formulé, est due à une sous-détermination de cet énoncé, au sens où deux prémisses essentielles: "Qut ment" et "Gui dit ta veritié" ne sont jamais données. Il s'ensuit que le paracioxe du Menteur provient d'un raisonnement dont les conditions de vérité riont pas étế suffisamment spécifiées.

De plus, la prise en compte de ces deux prémisses, qui a rendue possible la stabilisation du paradoze, nous permet de considérer que la loi de Leibniz a été respectée à tous les niveaux de langage dans notre résolution. En effet, la stabilisation des deux énoncés a été obtenue par le biais de substitutions conformes à la loi de Leibniz, et en tenant compte des interactions entre les prédicats de farsseté qui provoquaient des changementș de valeurs de vérité. Nous pouryons ainsi considérer qua'au niveau $\mathbf{n}+2$, les deux énoncés sont identiques, puisqu'ils peuvent être substitués l'un à l'autre sans que leur valeur de vérité soit altérée. Nous étions en effet en ptésence d'un énoncé qui était vaai, et après substitution de l'énoncé đun niveau n +1 dans cet énoncé, nous avons pu considérer que l'énoncé obtenu ếtait aussi vrai. II s'ensuit que la cause du non-respect de la loi de Leibniz par le paradoxe du Menteur, tel qu'il est habituellement formulé, est due à la sous-détermination de son contenu propositionuel.

Etant donné que nous avons montré qu'une résolution analogue peut être obtenue dans le cas du Paradoxe autoreférent indirect 1, la résolution dn paradoxe simpie ici exposée ainsi que la résolution du

1 CF. GODART, B., 1987. 
paradoxe autoréférent indirect nous permettent de déduire la règle de conditions de vérité suivante :

Lorsqu'un paradoxe sui-falsificatenr simple ou autoréférent indirect s'est engendré, il faut poser au niveau $n+1$ la prêmisse que " $\mathrm{x}$ est un menteur" sur la personne qui s'est présentée (cas du paradoxe simple) ou qui a été désignée (cas du paradoxe autoréférent indirect) comme étant le menteur. De plus, il faut placer au niveau $n+1$, la prémisse que " $\mathrm{x}$ dit la véritê" sur la personne qui confirme l'énoncé sui-falsificateur (cas du menteur simple) ou l'énoncé qui désigne la personne qui ment (cas du paradoxe autoréférent indirect).

Cette règle d'attribution de conditions de vérité, qui permet la stabilisation sếmantique et par conséquent la résolution des paradoxes de la famille du Menteur, a été obtenue dans le cadre d'une approche qui a respecté l'évolution thaturelle dynamique du paradoxe, en faisant en sorte que la cohérence des différents univers de croyance des protagonistes en présence (Socrate, Robert) soit respectée par le locuteur, lorsque celui-ci prenait en charge dans son propre univers de croyance les propositions tenues pour vraies par ces protagonistes. Nous avons en effet pu montrer, lors de notre étude du paradoxe du Menteur simple, qu'il était possible de sortir du dilemme âu à la présence de deux résolutions concurrentes, en mettant en évidence qu'une seule de ces résolutions avait pour fondement, à l'intérieur même de l'waivers de croyance du locuteur, un respect de l'évolution naturelle du paradoxe, car les conditions de vérité ajoutées par le locuteur permettaient de rendre compte fidèlement de la cohérence du discours de Robert, dont nous savions qu'elle reflétait exactement l'Évolution naturelle du paradoxe.

Aussi, étant donné que la version do paradoxe du Menteur de Buridan est réductỉble au paradoxe đu Menteur autoréférent direct sous la forme:

Socrate: Socrate dit faux. 
nous poavons en déduire que la résolution du paradoxe oủ Menteur sous cette dernière forme est la suivante:

Socrate s'est présenté comme étant un menteur, donc il faut placer la prémisse que "x est un menteur" devant l'érinoncé de Socrate. D'où :

\section{$F($ Socrate dit faux)}

Il s'ensuit que la signification de l'énoncé de Socrate est : Socrate dit vrai. Cetti signification est exempte de toute contradiction, et par conséquent ne présente plus aucume instabilité sémantique.

De plas, cette règle dattribution de conditions de vérité nous permet maintenant de mieux cemer le rapport entretenu par le paracoxe da Menteur avec le champ de la pragmatique. Cette règle nous rend, en effet, capable de déduire qu'un paradoxe sui-falsificateur simple "exploite" (au sens de P. Grice, 1979, p. 64) effectivement la maxime de qualité de P. Grice. Ainsi, dans le paradoxe du Menteur simple, la maxime de quantité est respectée, car le protagoniste s'est effectivement présenté comme étant un Meuteur. Cependant, ce respect de la maxime de quantitté permet une "exploitation" de la maxime de qualité, car le protagoniste peut de ce fait mentir, tout en disant la yérité, puisqu'il nous a prévenu qu"il était un menteur.

Nous tenons anssi à faire remarquer que l'approche de résolution du paradoxe du Menteur que nous avons proposée ne requiert pas la présence d'un locuteur omniscient pour que le paradoxe puisse être stabilisé. En effet, la procédure que nous avons établie exige seulement que le locuteur ait la propriété d'être logique, an sens où il doit être capable d'envisager tous les différents cas de figure compatibles avec la nature du problème qu'il a à résoudre. La sélection du cas de figure correspondant à la résolution đu paradoxe du Menteur s'effectue alors en prenant en considération, si deux résolutions concurrentes viennent à 
apparaître, la résolution qui rend compte le plus fídèlement de l'évolution naturelle du paradoxe.

Cette étude da paradoxe du Menteur entreprise dans le cadre de la Sémantique Naïve nous permet de proposer la définition suivante du Paradoxe du Menteur :

Paradoxe du Menters : Proposition engendrant un raisonnement de la forme d'une substitution leibnizienne, qui ne respecte pas la loi de Leibniz, car son contenu propositionnel est sous-déterminé.

La résolation du paradoxe du Menteur qui a été présentée, ainsi que la règle d'attribution de conditions de vérité qui a pu en être déduite, nous conduisent à penser que la résolution d'un paradoxe sui-falsificateur ne doit pas consister à rechercher, dans le raisonnement naturellement engenđré paz ce type de paradoxe, une prémisse qui serair fausse. II s'agit, en effet, pour résourdre un paradoxe sui-falsificateur, de mettre en évidence que le raisonnement naturellement engendré par ce type de paradoxe est, en fait, incomplet, au sens où une ou deux prémisses essentielles à l'élaboration mềme de ce raisonnement, et correspondant aux conditions de vérité du paradoxe, y sont absentes. I s'ensuit que la résolution d'un paradoxe sui-falsificatewr réside dans le fait qu'il fant restituer explicitement, à Iintérieur même đu raisonnement, la ou les prémisse(s) manquante(s), en fonction de la règle d'attribution de conditions de vérité.

La prise en considération de la ou des prémisses, dans le cadre d'une approche de résolution dynamique đu paradoxe, a pour conséquences que :

- l'evaluation du parađoxe ne conđạit plus au résultą que cet énoncé est faux si et seulement si il est vrai ;

- le calcul de la valeur de vérité de l'énoncé du Mentenr a pu être réalisé en logique binaire. 
Nous pouvons en déduire que le paradoxe du Menteur ne pose plus les problèmes :

- dinconsistance du langage naturel (contrairement à ce que pensait B. Russell) ;

- d'incomplétude du prédicat de vérité (contrairement à ce que pensait B. Van Fraassen).

Il s'ensuit que l'existence des paradoxes de la famille du Menteur au sein đu langage naturel ne peut être considérte comme nne prenve de 1'irréductibilité du langage naturel à un langage formel.

Béatrice GODART-WENDLING

\section{BIBLIOGRAPHIE}

GODART, B. (1987) "Les énonces Sui-falsificteteurs : nouvelle classe d'exceptions à Ia loi de Leibniz", Logique ei Analyse, Septernbre 1987, 119, pp. 235-256.

GOLDSTEIN, L. (1981) "Categories of Linguistic Aspects and Grelling"s Paradox", Linguistics and Philosophy, An international joumal, Austin, Volume 4, $\pi^{\circ} 3$, pp $405-421$.

HERZBERGER, H. (1982) "Naive Semantics and the Liar Paradox", The Journal of Philosophy, Volume LXXIX, $\pi^{\circ}$ 9, pp. 479-497.

MCCAWLEY, J.D. (1981) Everytining that Linguists Have always Wanted to Know about Logics (but were ashamed to ask), Oxford, Basil Blaciwell, $508 \mathrm{p}$.

MARTIN, R. (1983) Pour une Logique du Sens, Presses Universitaires de France, Paris, Collection : "Linguistique Nourvelle", 272 p.

MARTIN, R.E. (1967) "Toward a Solution to the Liar Paradox", The Philosophical Review, Yolume 76, pp. $279-311$.

MARTIN, R.L. (1970 \& 1978) "A Category Solution to the Liar", The Paradox of the Liar, Independance Ohio, Ridgeview Publishing Company, edited by RL. Martin, pp. 91-112.

RUSSELL, B.A.W. (1900) "Les paradoxes de la logique", Revue de Metaphysique et de Morale, Voinme 14, pp. 627-650.

RUSSELE, B.A.W. (1908) "Matherraticai Logic as Based on the Theory of Types", American Journal of Mathematics, Volume 30, pp. 222-262. Reprint in : Marsh, 1956, Logic and Knowledge, Essays of B. Russell, 1901-1950, Allen \& Unwin, pp. $57-102$ 
RUSSELL, B.A.W. (1943/69) Signification et Vérité (An Inquity into Meaning and Truth), Flammarion, Paris, $382 \mathrm{p}$.

VAN FRAASSEN, B.C. (I968) "Presupposition, Implication, and Self-reference", Journal of Philosophy, Volume 65 , pp. 136-152.

VAN FRAASSEN, B.C. (1970) "Inference and Self-reference", Synthese, 21, pp. 425-438. 